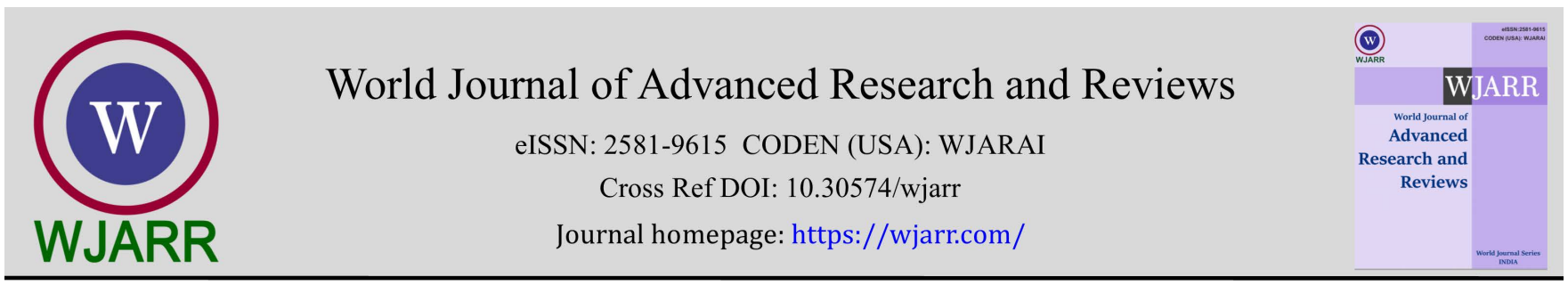

(REVIEW ARTICLE)

Check for updates

\title{
Software as a service and security
}

\author{
Hosniara Pervin * \\ Southeast University, Dhaka, Bangladesh.
}

World Journal of Advanced Research and Reviews, 2021, 11(03), 327-331

Publication history: Received on 15 August 2021; revised on 20 September 2021; accepted on 22 September 2021

Article DOI: https://doi.org/10.30574/wjarr.2021.11.3.0459

\begin{abstract}
Nowadays, Cloud Computing is an important hi-tech research area in the most recent innovation. These advancements give clients a few benefits: cost-adequacy, effective asset usage, cooperation, catastrophe recuperation, and elite. Cloud offers administrations for processing on a solitary mouse click. The overview was completed in the type of an online survey. This examination paper exhibits that SaaS is safer than other web applications. SaaS is superior to different applications and SaaS is savvy. This Research paper investigates the significance of SaaS for productive distributed computing for associations and its suggestions. This examination paper additionally talks about the significance of SaaS application engineering, usefulness, proficiency, benefits, and weaknesses.
\end{abstract}

Keywords: Cloud Computing; Access Control; Software as a Service; SaaS Architecture

\section{Introduction}

The ascent of Software-as-a-Service (SaaS) concurs with the ascent of cloud-based figuring. Distributed computing is the way toward offering innovation administrations through the web, which frequently incorporates information stockpiling, systems administration, and workers. Before SaaS was accessible, organizations hoping to refresh the product on their PCs needed to buy conservative plates containing the updates and download them onto their frameworks.

For enormous associations, refreshing programming was a tedious undertaking. After some time, programming refreshes opened up for download through the web, with organizations buying extra licenses instead of extra circles. Notwithstanding, a duplicate of the product actually should have been introduced on all gadgets that required admittance to it.

With SaaS, clients don't have to introduce or refresh any product. All things being equal, clients can sign in through the web or internet browser and interface with the specialist co-op's organization to get to the specific help.

SaaS is viewed to act as an illustration of endogenous development hypothesis, which is a monetary hypothesis that buys in to the conviction that financial development can be accomplished through growing new innovations and enhancements underway proficiency. Innovation organizations, monetary administrations organizations, and utilities have driven the business world in embracing SaaS innovation.

\footnotetext{
* Corresponding author: Hosniara Pervin

Southeast University, Dhaka, Bangladesh. 


\section{Key Security Requirements in Cloud Computing}

As indicated by NIST, privacy, accessibility and trustworthiness are a crucial need for distributed computing, similarly as some other data innovation the board framework. Further approval, verification, responsibility and security as cloud insurance models are presented, as found in Fig 2.

Classification implies keeping up the data of clients and permitting extraordinary people only for admittance to information.

Respectability implies a pledge to guarantee that data isn't balanced or changed as it is prepared or sent and that the methodology of purchasers to change, correct, rehash or eliminate data is simply permitted.

Validation includes guaranteeing the client's respectability when giving admittance to subtleties and this can be attainable with the utilization of such protections on their records.

Accessibility implies that the data recorded by the client or the administrations it needs is ceaselessly open any place and any place it is required.

Approval intends to ensure that the choices to get to it are apportioned for customers who have submitted uncommon subtleties.

\section{Related work}

SaaS offers an assortment of benefits over customary programming authorizing models. Since the product doesn't live on the authorizing organization's workers, there is less interest for the organization to put resources into new equipment.

It is not difficult to execute, simple to refresh and troubleshoot, and can be more affordable (or if nothing else have lower in advance expenses) since clients pay for SaaS as they go as opposed to buying numerous product licenses for various PCs [1].

SaaS has various applications, including:

- Email services

- Auditing functions

- Automating sign-up for products and services

- Managing documents, including file sharing and document collaboration

- Shared company calendars, which can be used for scheduling events

- Customer relationship management (CRM) systems, which is essentially a database of client and prospect information. SaaS-based CRMs can be used to hold company contact information, business activity, products purchased as well as track leads.

\section{Methodology}

In this part, we present my proposed a strategy that used to characterize and arrange the security designs in Cloud SaaS. As demonstrated in (Figure 1) Cloud security design definition measure, we tracked down the entire interaction into five stages from security prerequisites recognizable proof to security design arrangement in sync 5 . In each progression, existing rules might be utilized. For instance, security and hazard evaluation is led dependent on the OWASP rule [2].

The underlying advance of the system focuses on the security requirements for Cloud SaaS. We consider all the possible security necessities covering different points from data and system security to correspondence assurance and security. Our complete goal is to list all the security essentials needed for structure the protected, trust and legal consistence Cloud SaaS application. Concerning real necessities, we separate different data getting ready essentials (e.g., the work and obligation of data processor or individual data usage control) under the degree of GDPR. The aftereffect of this movement is an overall Cloud security plan (see Table 1) for SaaS application. These unusual state security essentials are then used for studying security and risk in Cloud SaaS [2]. 


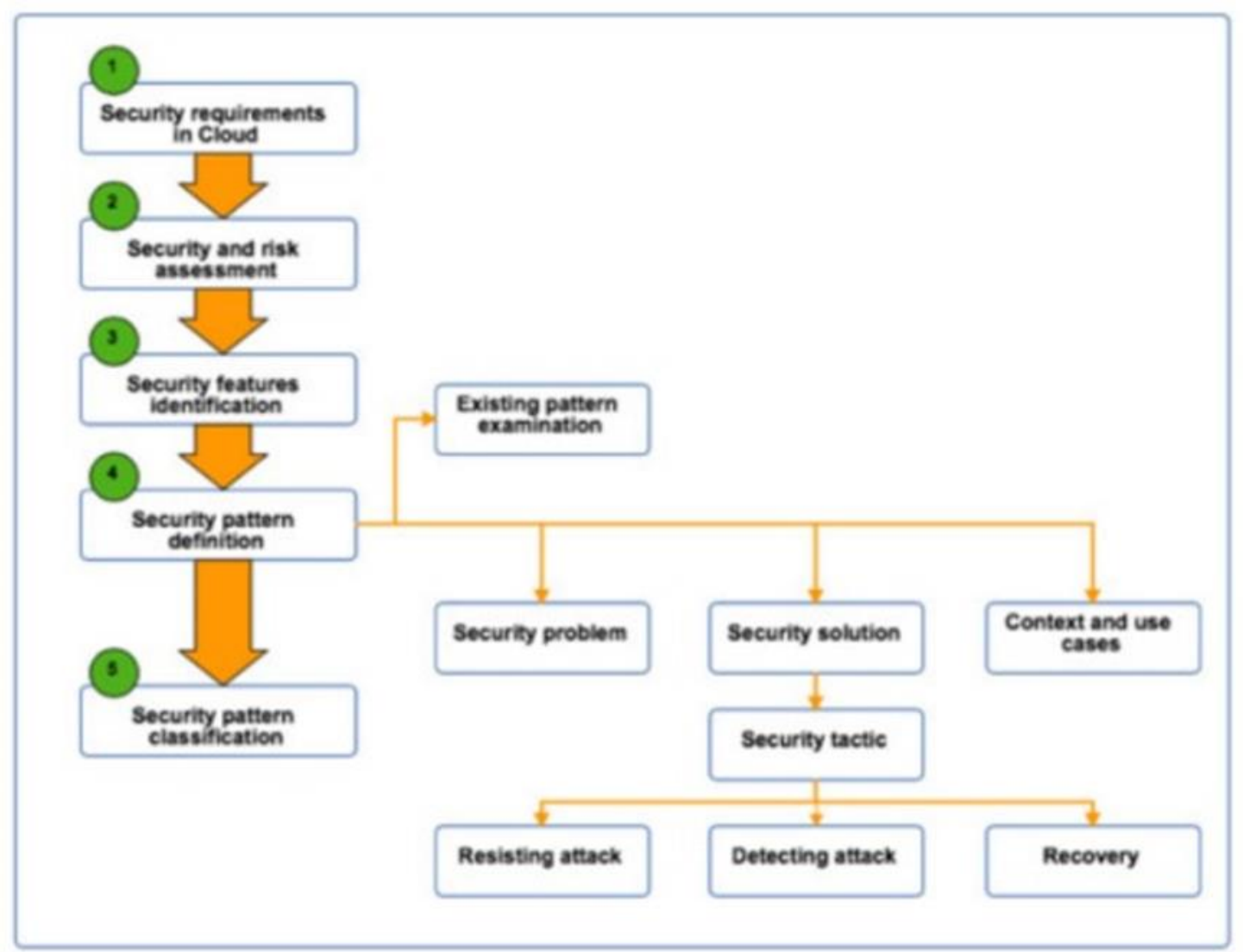

Figure 1 Cloud security pattern definition process

Table 1 Top-Level Cloud Security Requirements

\begin{tabular}{|l|l|}
\hline ID & Requirement Description \\
\hline R1 & Must provide protection to system's components \\
\hline R2 & Must be able to prevent unauthorized access and intrusion to system and resources \\
\hline R3 & Must be able to monitor networks requests \\
\hline R4 & Must have auditing option and be able to recover from a breach \\
\hline R5 & Must ensure data protection at rest and in transit \\
\hline R6 & Must ensure privacy protection and regulatory compliance \\
\hline R7 & Must provide secure communication between modules \\
\hline R8 & Must provide protection to system's resources. \\
\hline
\end{tabular}

\section{SaaS Challenges and Risks}

SaaS likewise represents some possible dangers and difficulties, as organizations should depend on external merchants to give the product, keep that product fully operational, track and report precise charging and work with a safe climate for the business' information.

Issues outside client ability to control. Issues can emerge when suppliers experience administration interruptions, force undesirable changes to support contributions or experience a security penetrate - all of which can profoundly affect the 
clients' capacity to utilize the SaaS offering. To proactively moderate these issues, clients ought to comprehend their SaaS supplier's SLA and ensure it is authorized. Clients let completely go over forming. In the event that the supplier embraces another form of an application, it will carry out to the entirety of its clients, whether or not or not the client needs the more current adaptation. This may require the association to give additional time and assets to preparing.

Trouble exchanging merchants. Similarly, as with utilizing any cloud specialist co-op, exchanging merchants can be troublesome. To switch merchants, clients should relocate a lot of information. Moreover, a few sellers utilize restrictive innovations and information types, which can additionally confound client information move between various cloud suppliers. Merchant lock-in is the point at which a client can only with significant effort progress between specialist coops because of these conditions [3]. Security. Cloud security is frequently referred to as a critical test for SaaS applications.

Fig. 4 Text Detection and Inpainting (a) Original image (b) Image after applying first set of criteria (c) Image after applying second set of criteria (d) Image mask (e) Painted image using patch size 5 x 5 and search window size 81 x 81 .

To eliminate small objects, connected component labelling is applied to the resultant image.(c) represents text detection by applying second set of criteria which eliminates all the objects whose area is less than 300 and filled area is less than 500.

\section{Research Model}

In the writing, the RBV hypothesis accentuates the significance of firm abilities in the production of firm advantages. Besides, as indicated by the serious technique hypothesis, an association's firm exhibition is impacted by the picked cutthroat system, which causes us to anticipate that its capacities, upheld by functionalities gave through SaaS EA, will build up the chosen business methodology to get an upper hand. Firm advantages ought to be considered in its inner and outside measurements, to be more precise regarding how firm capacities apply its belongings, a partition that has been ordered in operational and key advantages, that we assign as business worth and firm execution, and we are misusing how the last is affected by the previous. In like manner, we fostered an exploration model, as demonstrated in Fig. 2 [4].

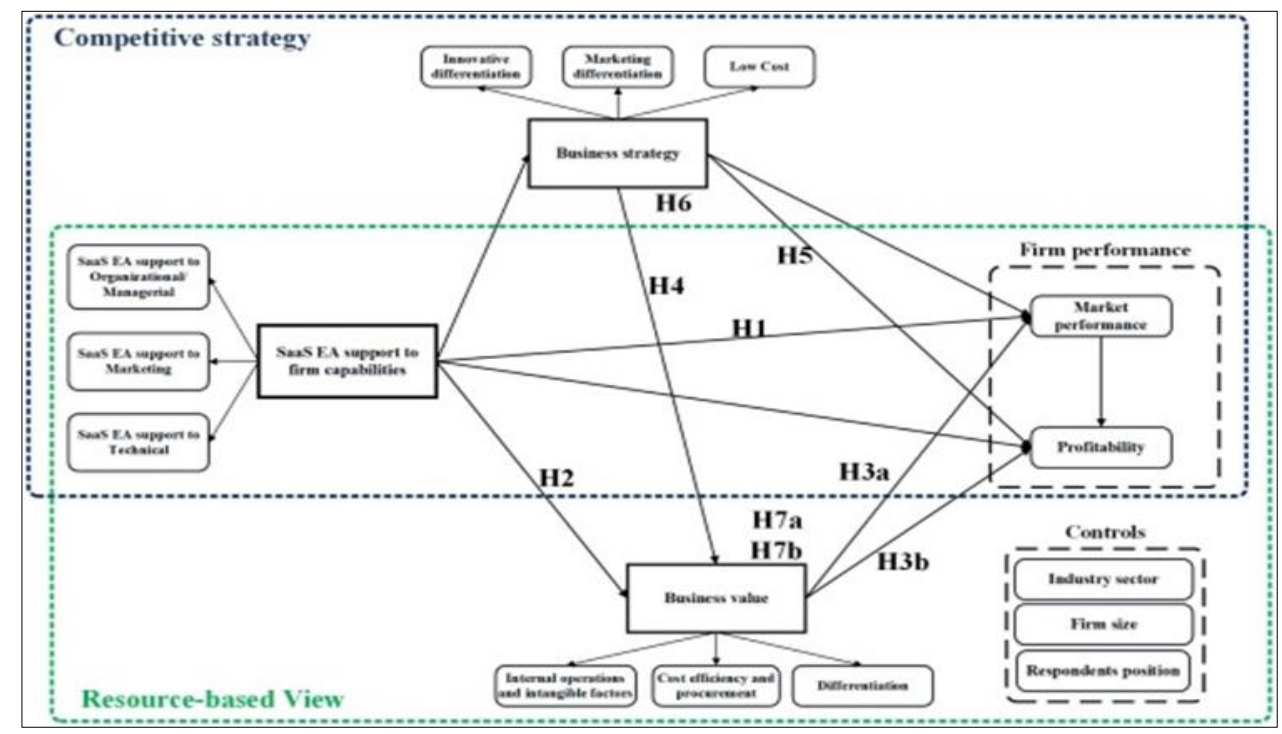

Figure 2 Research model

\section{Identifies Threats}

In the efforts to achieve efficient and effective neutralization of threats as well as reduction of false positives, firms collect and carry out analysis of log data from the Azure resources, where the network along with third-party solutions such as firewalls and endpoint solutions are implemented. Security centers analyze information, often correlating information from numerous sources in an attempt to uncover threats along with their intensity. Microsoft security scientists are continually vigilant for dangers. They approach a far-reaching set of telemetry picked up from Microsoft's worldwide nearness in the cloud and on-premises. This wide coming to and different gathering of datasets empowers 
Microsoft to find new assault examples and patterns over its on-premises customer and endeavor items, just as its online administrations. Subsequently, the Security Center can quickly refresh its identification calculations as aggressors discharge new and progressively complex endeavors. This methodology encourages you to keep pace with a quickmoving risk condition [4].

\section{Evaluation Technology}

The security testing strategy for distributed computing framework can be separated into four classes: security work, security ensure, and natural versatility and execution prerequisites as indicated by the security specialized necessities. As indicated by the necessities of every sort of framework, the testing strategies are concentrated separately. Security useful necessities are the particular prerequisites of distributed computing security capacity of data security items, the particular prerequisites of distributed computing data security items incorporate personality verification, access control, security the executives, can't sidestep, against assault, review and arrangement information assurance, activity observing, flexible versatility and virtual asset security to guarantee security; Security ensure prerequisites are advanced explicit necessities for distributed computing data security items in the turn of events and utilization of the substance of the report, like design the board, conveyance and activity, improvement measure, direction records and life cycle support; natural flexibility necessities are advanced for the particular necessities of the application climate of distributed computing data security items, for example, IPv6 ecological versatility; the exhibition necessities is to make the presentation file of distributed computing framework and distributed computing items meet the prerequisites Fixed, for example, virtual machine I/O time, assault sifting and protection execution, and so on What's more, the weakness of the working framework level of distributed computing framework and gear is broke down and exhumed to shape an assault test set [7].

\section{Conclusion}

At long last, I arrived at a resolution dependent on Secure Development, Operation and Administration, Privacy and Confidentiality, Secure Architecture, Compliance and administrative, Identification, Authentication and Authorization that could base Software as an assistance's security is more grounded than other kind of utilization. The Software as a Service (SaaS) model offers customers essential benefits, for instance, improved operational adequacy and decreased costs. Regardless, to crush customer stresses over application and data security, traders should address these issues head-on.

\section{Compliance with ethical standards}

\section{Acknowledgments}

This Research paper investigates the significance of SaaS for productive distributed computing for associations and its suggestions.

\section{References}

[1] M. Grant. Software-as-a-Service (SaaS), in Investopedia. 2 Feb 2020.

[2] MHU Shairf, R Datta. Software as a Service has Strong Cloud Security", International Journal of Research in Engineering and Management. 2019; 1(2): 18 - 27.

[3] W Chai, K Casey. Software as a Service (SaaS), Search Cloud Computing. February 2021.

[4] MHU Shairf, R Datta. Threat Modeling Stapes to Mitigate Could Threat. International Journal of Computer \& Organization Trends (IJCOT). May - June 2020; 10(3).

[5] J Rodrigues, P Ruivo, T Oliveira. Mediation role of business value and strategy in firm performance of organizations using software-as-a-service enterprise applications", Information \& Management. January 2021; 58(1): 103289.

[6] F Thabit, S Alhomdy, HA Al-Ahdal, S Jagtap. A new lightweight cryptographic algorithm for enhancing data security in cloud computing, Global Transitions Proceedings. June 2021; 2(1): 91-99.

[7] HH Song. Testing and Evaluation System for Cloud Computing Information Security Products, Science Direct, Procedia Computer Science. 2020; 166: 84-87. 